\title{
Demyelinating disorder of the central nervous system occurring in black South Africans
}

\author{
G Modi, A Mochan, M Modi, D Saffer
}

\begin{abstract}
Objectives-To investigate the nature and cause in eight black South African patients of a recurrent (multiphasic), remitting, and relapsing demyelinating disease of the CNS.

Methods-The clinical and laboratory investigations and radiological manifestations of these patients were documented.

Results-Each patient had two or more acute attacks of demyelinating disease affecting the CNS. The clinical presentations of the patients were predominantly those of multiphasic neuromyelitis optica (NMO). Brain MRI in these patients showed features consistent with those described for acute disseminated encephalomyelitis (ADEM), as well as lesions that are described in multiple sclerosis. There was involvement of the corpus callosum in addition to typical ADEM lesions. Laboratory investigations excluded all other known causes of multiphasic CNS demyelination. Oligoclonal antibodies were not detected in these patients at any time. The patients were all from a population with a low risk for MS (black South Africans). Conclusion-The patients described here represent a new phenotypic expression of a recurrent (multiphasic), steroid sensitive, inflammatory demyelinating disorder of the CNS occurring in black South Africans. The disorder is either a distinct inflammatory demyelinating disorder of the CNS of as yet unknown aetiology, or a varied form of MS (ADEM/NMO) occurring in a population with a low risk (where the genetic trait and environmental risk factors for MS do not exist) for MS. (F Neurol Neurosurg Psychiatry 2001;70:500-505)
\end{abstract}

Department of

Medicine, Chris Hani

Baragwanath Hospital,

Faculty of Health

Sciences, University of

the Witwatersrand,

Johannesburg, South

Africa

G Modi

A Mochan

D Saffer

Department of

Radiology

M Modi

Correspondence to:

Professor G Modi, Box 909,

Lenasia 1820, South Africa

gmodicms@mweb.co.za

Received 5 June 2000 and in revised form

16 October 2000

Accepted 6 November 2000 forms include relapsing-progressive, primary progressive, and progressive-relapsing.

Acute disseminated encephalomyelitis $(\mathrm{ADEM})$ is a rare multifocal, inflammatory demyelinating disorder of the CNS. It occurs after vaccination against various viruses, follows on many viral infections (specific or nonspecific) and can also occur spontaneously. ${ }^{67}$ There is no specific age, sex, or race predilection. It is most commonly a non-progressive acute monophasic illness. ${ }^{8}$ The clinical manifestations are widespread CNS disturbances, including coma, drowsiness, seizures, and multifocal neurological signs due to involvement of the brain, spinal cord, and optic nerves. ${ }^{8} \quad$ Para/tetraparesis/plegia, ascending sensory dysfunction, segmental sensory level, neurogenic bladder, and altered cerebral function are the dominant clinical features. ${ }^{9}$ The diagnosis is made when there is an antecedent infection followed by focal neurological disturbances, an abnormal CSF analysis (increased protein with cells), and where available, MRI evidence of multifocal white matter involvement. The treatment of choice is high dose intravenous corticosteroids. ${ }^{10}$ The demyelination in ADEM is self limiting. Serial MRI of patients have shown resolution of the lesions. ${ }^{811}$ Repeated episodes are rare. ${ }^{811}$

Neuromyelitis optica (NMO), also known as Devic's disease, is an inflammatory demyelinating disease characterised by a severe acute transverse myelitis with bilateral simultaneous or sequential optic neuropathy, resulting in paraplegia and blindness with or without recovery. ${ }^{12}{ }^{13}$ There is no clinical involvement beyond the optic nerves and spinal cord. ${ }^{12}{ }^{13}$ The course may be monophasic or multiphasic. ${ }^{12} 13$ Patients with a monophasic course present with rapidly sequential index events (median 5 days) and show moderate recovery. ${ }^{13}$ Patients with a relapsing or multiphasic course have a median interval of 166 days between the index events. This is followed within 3 years by clusters of severe relapses isolated to the optic nerves and spinal cord. ${ }^{13}$ Patients with relapsing NMO show poor recovery and develop stepwise disability. ${ }^{12}{ }^{13}$ Neuromyelitis optica is said to occur more commonly in non-white race groups. $^{3}$

We describe here eight black South African patients with a recurrent (multiphasic) and relapsing inflammatory demyelinating disorder of the CNS, with clinical, laboratory and radiological features encompassing aspects of MS, NMO, and ADEM.

\section{Patients and methods}

The eight patients described here in table 1 were seen at the Chris Hani Baragwanath hospital, in Soweto, South Africa, between the 
years 1996 and 2000. The $\mathrm{CHBH}$ is a 3300 bed public University Hospital that serves a predominantly black urban population of about 3 million people.

The diagnosis of inflammatory demyelination was based on MRI and CSF analysis. The patients were diagnosed as recurrent/ multiphasic or relapsing on the basis of a new or different clinical manifestation occurring after an initial "attack". The presence or absence of antecedant infections was documented with each attack.

All patients were subjected to detailed blood and CSF investigations to exclude vasculopathy, syphilis, sarcoidosis, tuberculosis and other causes of CNS demyelination. Patients had a full blood count, erythrocyte sedimentation rate, urea, and electrolytes, prothrombin index, blood glucose, Wasserman reaction, liver function tests, serum protein electrophoresis thyroid function tests, serum vitamin B12, and folate concentrations, HIV, HTLV-I, antinuclear factor, serum angiotensin converting enzyme, serum immunoglobulins, and bilharzia CFT. Analysis of CSF included bloodbrain barrier studies, oligoclonal antibody analysis, microscopy, culture, tuberculosis polymerase chain reaction and enzyme linked immunosorbent assay, biochemistry, and cell count.

Table 1 Course, outcome, and clinical profiles of the patients described in the text

\begin{tabular}{|c|c|c|c|c|}
\hline & Case 1 & Case 2 & Case 3 & Case 4 \\
\hline Age (y) & 42 & 37 & 31 & 29 \\
\hline Sex & Male & Male & Female & Female \\
\hline Preceding illness & GIT & Nil & Respiratory & Nil \\
\hline First attack & Hemiplegia & T4 Level & C2 Myelitis & Optic neuritis \\
\hline CSF & ND & Increased protein & Increased protein & Normal protein \\
\hline MRI-WML & ND & $\begin{array}{l}\text { Cells, no OCB } \\
\text { ND }\end{array}$ & $\begin{array}{l}\text { Cells, no OCB } \\
\text { Lower medulla } \\
\text { Upper cervical }\end{array}$ & $\begin{array}{l}\text { No cells, no OCB } \\
\text { ND }\end{array}$ \\
\hline Therapy & Nil & Nil & iv Solumedrol & Nil \\
\hline Outcome & Full recovery & Poor & Ambulatory & Partial recovery \\
\hline Interval & 2 Years & 2 Months & 5 Months & 2 Months \\
\hline Second attack & T6 Myelitis & Optic neuritis & Optic neuritis & C4 Myelitis \\
\hline CSF & $\begin{array}{l}\text { Normal protein } \\
\text { Cells, no OCB }\end{array}$ & ND & ND & $\begin{array}{l}\text { Increased protein } \\
\text { Cells, no OCB }\end{array}$ \\
\hline MRI-WML & Diffuse PV, CC & PV, CS, CR, CC & ND & Lower medulla to \\
\hline Therapy & $\begin{array}{l}\text { Pontine, thoracic cord atrophy } \\
\text { iv Solumedrol }\end{array}$ & $\begin{array}{l}\text { Patchy thoracic } \\
\text { iv Solumedrol }\end{array}$ & Nil & $\begin{array}{l}\text { T1 elongated lesion } \\
\text { iv Solumedrol }\end{array}$ \\
\hline Outcome & Poor & Good & Good & Ambulatory \\
\hline Interval & & & 2 Months & \\
\hline Third attack & & & T6 Myelitis & \\
\hline \multirow[t]{2}{*}{$\mathrm{CSF}$} & & & Increased protein & \\
\hline & & & Cells, no OCB & \\
\hline \multirow[t]{2}{*}{ MR -WML } & & & Normal brain & \\
\hline & & & Mid-thoracic & \\
\hline \multirow{3}{*}{$\begin{array}{l}\text { Therapy } \\
\text { Outcome }\end{array}$} & & & iv Solumedrol & \\
\hline & & & Poor & \\
\hline & Case 5 & Case 6 & Case 7 & Case 8 \\
\hline Age (y) & 41 & 8 & 33 & 31 \\
\hline Sex & Female & Female & Female & Female \\
\hline Preceding illness & Nil & Nil & GIT & Nil \\
\hline First attack & Optic neuritis & Myelitis & Bulbar & Optic neuritis \\
\hline CSF & $\mathrm{ND}$ & ND & $\begin{array}{l}\text { Increased protein } \\
\text { No cells, no OCB }\end{array}$ & $\begin{array}{l}\text { Normal protein } \\
\text { No cells, no OCB }\end{array}$ \\
\hline MRI-WML & ND & ND & PV, medulla & ND \\
\hline Therapy & Nil & Nil & iv Solumedrol & iv Solumedrol \\
\hline Outcome & Good & Good & Good & Good \\
\hline Interval & 5 Months & 8 Months & 18 Months & 16 Months \\
\hline Second attack & Craniocervical & Optic neuritis & Optic neuritis & Optic neuritis \\
\hline CSF & $\begin{array}{l}\text { Increased protein } \\
\text { No cells, no OCB }\end{array}$ & ND & $\begin{array}{l}\text { Increased protein } \\
\text { Cells, no OCB }\end{array}$ & ND \\
\hline MRI & $\begin{array}{l}\text { PV, CR, CS } \\
\text { Midbrain, left cerebellum } \\
\text { C1 to T1 elongated lesion }\end{array}$ & PV & ND & ND \\
\hline Therapy & iv Solumedrol & Oral steroids & iv Solumedrol & Nil \\
\hline Outcome & Ambulatory & Poor & Poor & Poor \\
\hline Interval & & 9 Months & 1 Month & 10 Months \\
\hline Third attack & & Craniocervical & C4 Myelitis & C2 Myelitis \\
\hline CSF & & $\begin{array}{l}\text { Normal protein } \\
\text { No cells, no OCB }\end{array}$ & ND & $\begin{array}{l}\text { Increased protein } \\
\text { Cells, no OCB }\end{array}$ \\
\hline \multirow[t]{2}{*}{ MRI-WML } & & CC, subcortical & PV, CS & PV, CR, CS \\
\hline & & $\begin{array}{l}\text { Midbrain } \\
\text { Cervicomedullary }\end{array}$ & C2 to C6 Lesion & $\mathrm{C} 1$ to $\mathrm{C} 6$ lesion \\
\hline Therapy & & iv Solumedrol & iv Solumedrol & iv Solumedrol \\
\hline Outcome & & Ambulatory & Poor & Good \\
\hline Interval & & & 12 Months & \\
\hline Fourth attack & & & C2 Myelitis/OPN & \\
\hline \multirow[t]{2}{*}{ CSF } & & & Increased protein & \\
\hline & & & Cells, no OCB & \\
\hline \multirow[t]{2}{*}{ MRI-WML } & & & $\mathrm{CC}$ & \\
\hline & & & $\mathrm{CCJ}$ to $\mathrm{C} 4 / 5$ lesion & \\
\hline Therapy & & & iv Solumedrol & \\
\hline Outcome & & & Poor & \\
\hline
\end{tabular}

$\mathrm{OPN}=$ Optic neuritis; $\mathrm{CCJ}=$ craniocervical junction myelitis; $\mathrm{WML}=$ white matter lesions; $\mathrm{CC}=$ corpus callosum; $\mathrm{CR}=$ corona radiata; $\mathrm{CS}=$ centrum semiovale; $\mathrm{PV}=$ periventricular $\mathrm{OCB}=$ oligoclonal antibody bands; $\mathrm{GIT}=$ gastrointestinal tract illness; $\mathrm{C}=$ cervical level; $\mathrm{T}=$ thoracic level; iv=intravenous. The iv steroid used in all patients was methylprednisolone (solumedrol). 
Radiological studies included chest radiographs, myelograms, CAT (computed axial tomography) of the brain, gallium scans and MRI of the brain and spinal cord. Brain scans were obtained using a Siemens 1.0T superconducting magnet. T1, T2, FLAIR (fluid attenuated inversion recovery) and proton density sequences were obtained on all patients. Chest radiographs and gallium scans were were normal in all patients.

\section{ILLUSTRATIVE CASE HISTORIES}

Case 3

A 31 year old black woman presented with acute paralysis of all four limbs after a flu-like illness. She had respiratory distress and required ventilation and treatment in the intensive care unit. She was incontinent for urine and faeces. Clinical examination disclosed flaccid tetraplegia with increased reflexes and an ill defined sensory level at $\mathrm{C} 2 / 3$.

Nerve conduction tests were normal. studies on CSF showed a protein content of $0.69 \mathrm{~g} / 1$ with 50 lymphocytes and 2 neutrophils. Glucose concentration was $3.8 \mathrm{mmol} / \mathrm{l}$. A tensilon test was negative.

Blood investigations were normal or negative. Evoked potentials were not done. During her stay in the intensive care unit her tone began to increase. An MR scan of the brain and cervical spine showed increased signal intensities on T2 weighted images in the upper cervical cord and lower medullary regions of the brain stem. Moderate enhancement of these lesions was noted with intravenous gadolinium. The remainder of the brain examination was normal. She was treated with intravenous methylprednisolone ( $1 \mathrm{~g}$ daily for 5 days). This was followed by oral prednisone at a dose of 60 mg daily. She improved rapidly and recovered respiratory function within 3 weeks.

She was then transferred to the neurology ward. Her steroids were gradually tapered. She recovered almost completely and at the time of discharge was walking unaided. She remained in hospital for 3 months. She was readmitted 1 month later for treatment of severe lower limb cramps. Her tone was increased in all four limbs and she had brisk reflexes. The cramps were treated with baclofen. She was still fully ambulatory.

A month after this second admission, she developed acute onset of pain and blindness in her right eye. She could not be admitted for investigations at that stage due to her social circumstances. No treatment was given, but she improved spontaneously. Two months after this event she developed acute paralysis of the lower limbs. She was found to have a flaccid areflexic paralysis with a T6 sensory level. She had complete incontinence for urine and faeces. In her right eye she had optic atrophy with a deafferented pupil. Her left eye was normal. She had no brain stem or cerebellar signs.

All the blood tests were repeated and were normal or negative. The CSF showed a protein content of $0.79 \mathrm{~g} / 1$ with 6 lymphocytes. Oligoclonal bands were absent. An MR scan of the brain and thoracic spine was obtained. The brain MRI was normal. The optic nerves were not well visualised. The MRI of the thoracic spine showed increased signal intensity on T2 weighted images in the mid-thoracic region. The cervical lesions seen previously had resolved. Evoked potentials were not investigated.

She was treated with intravenous methylprednisolone again. She remained paraplegic and incontinent.

\section{Case 6}

An 8 year old black girl presented with a history of having developed a sudden paraplegia in September 1997. She was diagnosed as having a viral myelitis at a provincial hospital. No specific investigations were done at the time and she was treated with physiotherapy. She recovered fully. In April 1998, she developed sudden onset of painless loss of vision in both eyes. She was again seen at the same provincial hospital. No investigations were done. She was treated with oral prednisone at a dose of $30 \mathrm{mg}$ daily for a week. She did not recover fully. She was then seen several months later by the paediatric department at our hospital. She was found to have bilateral optic atrophy. Detailed investigations disclosed no underlying treatable cause. An MR scan of the brain showed scattered subcortical and periventricular white matter lesions on the T2 and FLAIR sequences. There were no lesions in the corpus callosum, midbrain, or the cervicomedullary junction. A lumbar puncture was not done. No further treatment was considered at this stage.

In January 1999, she presented with a history of a sudden onset of flaccid tetraplegia with respiratory and bulbar paralysis. This followed a respiratory tract infection 1 week earlier. She was admitted to the intensive care unit and required ventilation. The clinical examination disclosed bilaterally deafferented pupils with optic atrophy and pendular nystagmus. The bulbar cranial nerves were paralysed, with an absent gag reflex. Her tone was flaccid in all four limbs with areflexia. The plantar responses were extensor. Sensation for all modalities was normal. Blood investigations were normal or negative. The CSF at this time showed a protein of $0.3 \mathrm{~g} / 1$ with no cells and a normal sugar and chloride concentration. There were no oligoclonal antibodies present. An MRI of the brain and spinal cord showed increased signal intensities in the cervicomedullary junction and in the midbrain, as well as involving the corpus callosum and subcortical white matter on T2 and FLAIR sequences. She was treated with intravenous methylprednisolone at a dose of $2 \mathrm{mg} / \mathrm{kg} /$ day for 5 days, followed by oral prednisone in tapering doses. She recovered bulbar function and was weaned off the ventilator after 10 days. She steadily recovered motor functions over a period of 6 weeks and could walk independently. As the motor functions improved the spasticity in her limbs became obvious. Her vision did not improve.

\section{Case 7}

A 33 year old black woman presented with bulbar weakness (dysphagia and dysarthria) and flaccid tetraparesis. These symptoms began 1 week after a gastrointestinal illness. Her mental 
state was normal. The cranial nerve examination disclosed a bulbar palsy with reduced palatal movements, decreased gag reflex, an absent jaw jerk, and a right Vth nerve sensory loss to pinprick. There were right sided cerebellar signs and upbeat nystagmus. The tone was reduced with brisk reflexes and extensor plantar responses. Her sensation was normal. Brain CT was normal. Blood investigations were normal or negative. Studies on CSF disclosed a protein content of $0.60 \mathrm{~g} / 1$ with no cellular response. Oligoclonal antibodies were absent. Evoked potentials were not done. Brain MRI showed hyperintense signals in the periventricular white matter and a hyperintense lesion in the medulla on T2 weighted images. She was treated with intravenous methylprednisolone ( $1 \mathrm{~g}$ daily for 5 days). She then received oral steroids in tapering doses over the next 3 weeks. Her bulbar functions improved within 2 weeks. She then developed increased tone in her limbs and regained power gradually over 6 weeks. At the time of discharge 3 months later, she was ambulatory, with normal bulbar functions, normal upper limbs, and mildly spastic lower limbs.

Eighteen months later she was admitted to hospital for a gastrointestinal illness. During this admission she developed sudden painless loss of vision in her right eye. Clinically she had optic neuritis with an afferent pupil defect and papillitis. Brain CT was normal. Blood investigations for bacillary and parasitic dysentries were normal. The HIV, Wassermann reaction, liver function tests, and immunoglobulins were normal. Stools were examined and showed no specific infections. Studies on CSF disclosed a protein content of $0.45 \mathrm{~g} / 1$ with 39 lymphocytes and normal glucose concentration. Oligoclonal antibodies were not present and blood-brain barrier studies were normal. She was treated with intravenous methylprednisolone ( $1 \mathrm{~g}$ daily for 5 days). She did not recover visual function of her right eye. Four weeks later, while in hospital, she developed a flaccid tetraparesis over 2 to 3 days. This began in the lower limbs and ascended to affect both upper limbs. Clinically, she was flaccid but with increased reflexes in all four limbs. The plantar responses were extensor. Respiratory and sphincter functions were preserved. There was a sensory level to all modalities at C4. An MRI of the cervical spine disclosed an elongated hyperintense lesion extending from $\mathrm{C} 2$ to C6 on the T2 weighted images. There was corresponding swelling of the spinal cord. Multiple foci of hyperintensity were noted in the subcortical white matter, involving the centrum semiovale in the T2 weighted images. The periventricular lesions seen previously were still present. The medullary lesion had disappeared. The right optic nerve showed increased signal intensity in the T2 sequences. She was treated again with intravenous methylprednisolone (I g daily for 5 days). This was changed to oral steroids in tapering doses over the next 3 weeks. Her recovery was poor but she became ambulatory. Twelve months later she presented again with flaccid tetraparesis and bilateral simultaneous loss of vision. Clinically she had a C2 myelitis with bilateral optic neuropathies. All investigations were repeated and were negative. The CSF showed a protein of $0.6 \mathrm{~g} / 1$ with 15 lymphocytes, no polymorphs or red cells. Oligoclonal antibodies were not detected. An MR scan of the brain and cervical spinal cord showed increased signal intensities in the craniocervical cord extending to $\mathrm{C} 4 / 5$ level. The lesion was scattered in appearance and not as homogenous and sausage shaped as seen previously. The brain MRI showed a striking ovoid lesion on the FLAIR sequence in the posterior part of the corpus callosum extending into the occipital radiation. Most of the subcortical lesions seen previously had disappeared. She was again treated with pulsed intravenous methylprednisolone (1 g daily for 5 days). This was changed to oral steroids in tapering doses over the next 3 weeks. Her response was poor. She is now wheelchair bound with poor visual acuity in both eyes.

\section{Results and discussion}

The profiles of the patients described here are as follows:

- Black African (no mixed ancestry)

- Female preponderance

- Average age 28.5 years

- Two or more different acute attacks of a disseminated disorder separated in time and space.

- MRI evidence of white matter lesions involving the periventricular areas; corpus callosum; brainstem; spinal cord

- CSF analysis indicating inflammatory demyelination; raised protein with pleocytosis; absence of oligoclonal antibodies

- Variable response to intravenous steroids

- Generally poor outcome with stepwise disability after repeated attacks

- Exclusion of all known causes of multifocal CNS demyelination.

The clinical course in these patients is as follows: (table 1)

Case 1-A hemiplegia followed 2 years later by a spastic myelopathy.

Case 2-A spastic myelopathy followed by a left optic neuritis 5 months later.

Case 3-A flaccid tetraplegia followed 5 months later by bilateral optic neuropathy. and a T6 myelopathy 2 months thereafter.

Case 4-Bilateral optic neuropathy followed by a tetraplegia 2 months later.

Case 5-Bilateral optic neuropathy followed by a craniocervical myelopathy 5 months later. Case 6-Transverse myelopathy followed 8 months later by bilateral optic. neuropathy and 9 months thereafter by a cervicomedullary myelopathy.

Case 7-A brainstem encephalomyelitis followed 18 months later by a unilateral optic neuritis, 1 month thereafter by a C4 myelopathy, and 12 months later by a C2 myelitis with bilateral optic neuropathy.

Case 8-Unilateral optic neuropathy followed 16 months later with bilateral optic neuropathy and 10 months thereafter by a $\mathrm{C} 2$ myelitis.

They essentially constitute a group of patients who had repeated attacks of an inflammatory disseminated demyelinating disorder of the CNS. The predominant clinical involvement of the optic nerves and spinal cord is in keeping with NMO. Only patients 1 and 7 
A

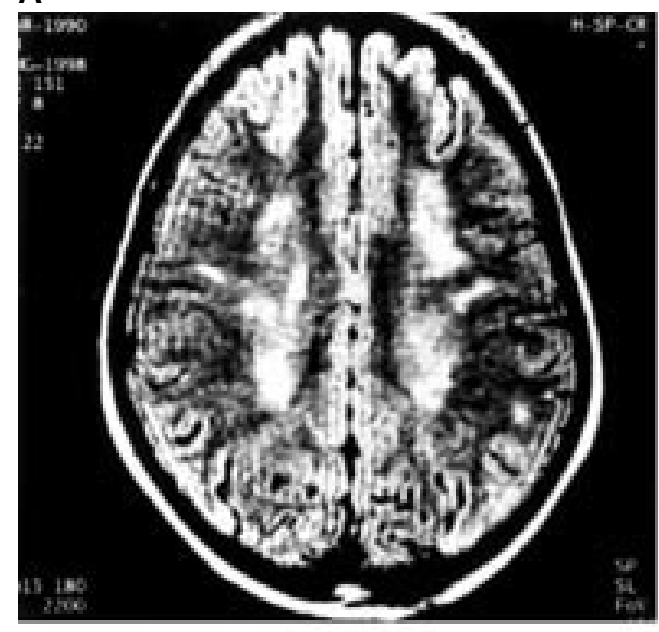

C

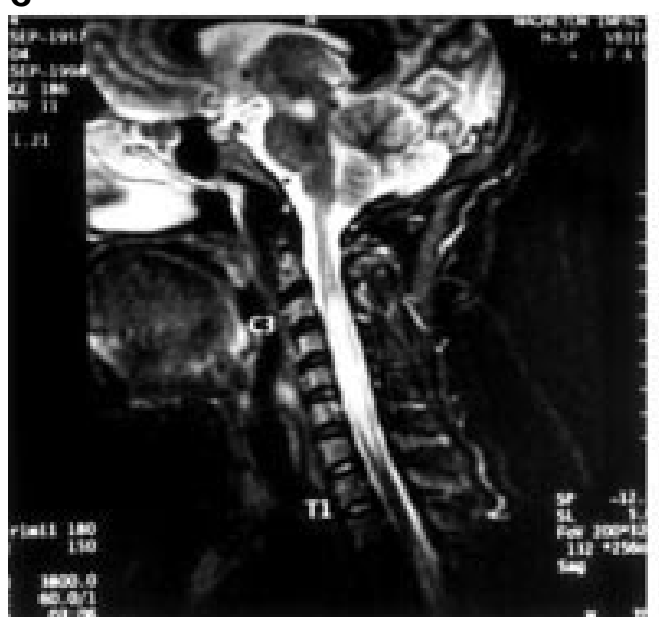

B

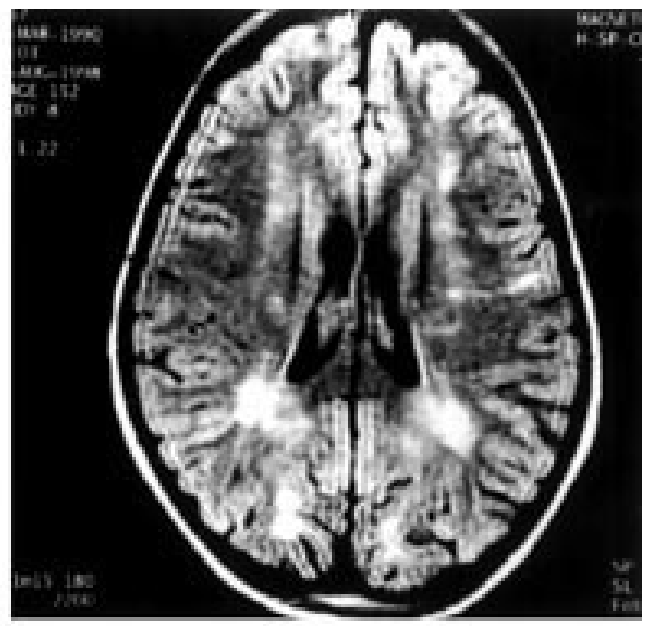

D

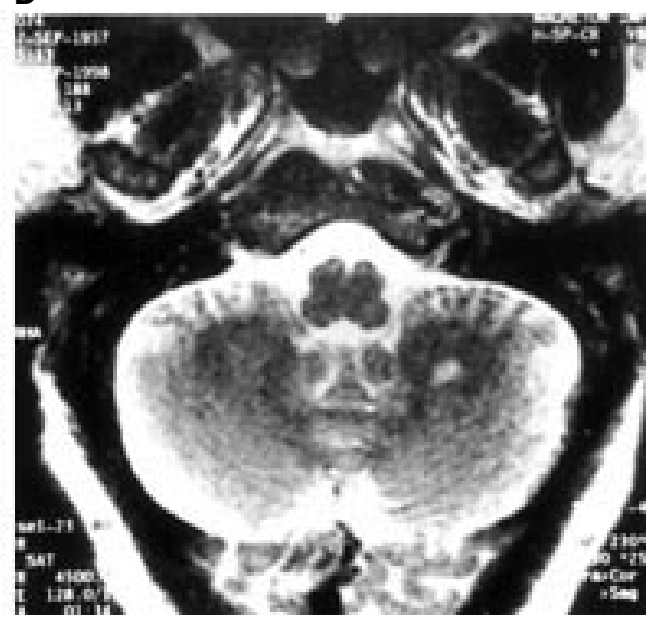

Figure 1 Illustrative MRI of the brain and cervical spinal cord (from case 5) showing widespread multifocal white matter hyperintensities on FLAIR ( $A$ and $B$ ) and T2 weighted sequences. The lesions involve the centrum semiovale with finger-like projections extending to the cortex, $(A)$ left parietal white matter; $(B)$ corona radiata and periventricular and occipital white matter; (C) midbrain and C3 to TI cervical spinal cord; (D) left cerebellar hemisphere.

had features outside the clinical range of NMO. The spinal cord lesions on MRI are also as those described in NMO, extending over several segments. ${ }^{12}$ Oligoclonal antibodies were not present in our patients. The CSF analysis did not disclose the presence of polymorphonuclear cells except in case 3 after the first attack. In NMO, CSF polymorphonucleocytosis is described to occur more commonly than in MS or ADEM. ${ }^{12}{ }^{13}$ Blood analysis in our patients did not show the presence of autoantibodies as is described to occur in NMO. ${ }^{12} 13$ The most important difference between NMO and our cases is the disseminated nature of the central white matter demyelination. In NMO the disease does not extend beyond the optic nerves and the spinal cord. ${ }^{12} 13$ The initial brain MRI in patients with NMO is normal and only occasionally on serial brain MRI are white matter lesions seen. These are described as scattered lesions, discrete and similar to unidentified bright objects. ${ }^{12}{ }^{13}$ In our cases the disseminated lesions are large and striking in appearance from the onset of the disease (figs $1,2,3$ ).

With respect to ADEM, the patients described here have: (1) a disseminated encephalo- myelitis as evidenced on MRI imaging; (2) antecedant non-specific infections in some cases; (3) bilateral optic nerve involvement; (4) CSF showing the presence of an increased protein with lymphocytosis; (5) absence of oligoclonal antibodies; (6) imaging features as described in ADEM - namely, large globular lesions in the brain and spinal cord, elongated spinal lesions extending over several segments; and (7) multiphasic course with new lesions on MRI after a new attack, ${ }^{14}$ as well as resolution of old lesions.

Against the diagnosis of ADEM is the presence of periventricular lesions, involvement of the corpus callosum, centrum semiovale, and callososeptal lesions (Dawson's fingers, fig 3).

In terms of MS, we argued against this diagnosis for the following reasons: the optic neuritis in MS is usually unilateral and the myelopathy is often partial. ${ }^{8}$ In our patients the optic neuropathies were bilateral (except for cases 2,7 , and in the first attack in case 8) and the myelopathies were complete (as is described for ADEM or NMO). The CSF findings in MS include a moderate but not striking pleocytosis with oligoclonal IgG bands. In our patients oligoclonal antibodies were not present even during the acute events. The pleocytosis, when 


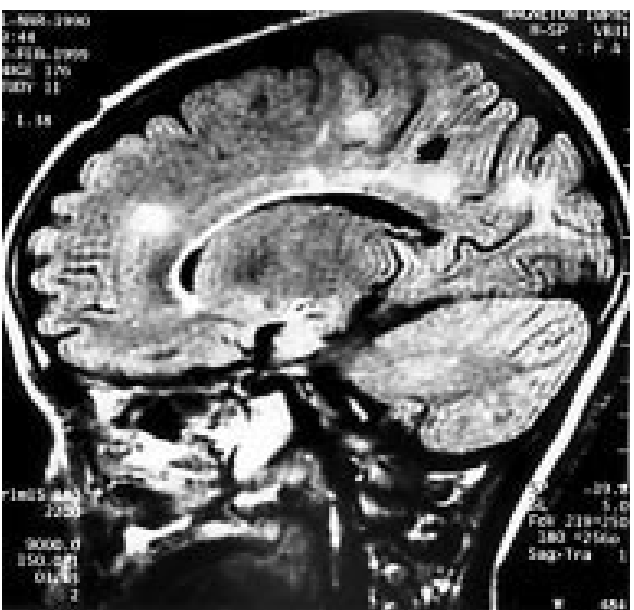

Figure 2 Illustrative MRI of the brain (case 6) showing multiple hyperintensities on FLAIR sequences involving the corpus callosum and subcortical white matter. The corpus callosum lesions are seen to extend into the centrum semiovale (arrow) resembling Dawson's fingers. The subcortical lesions are discrete and rounded.

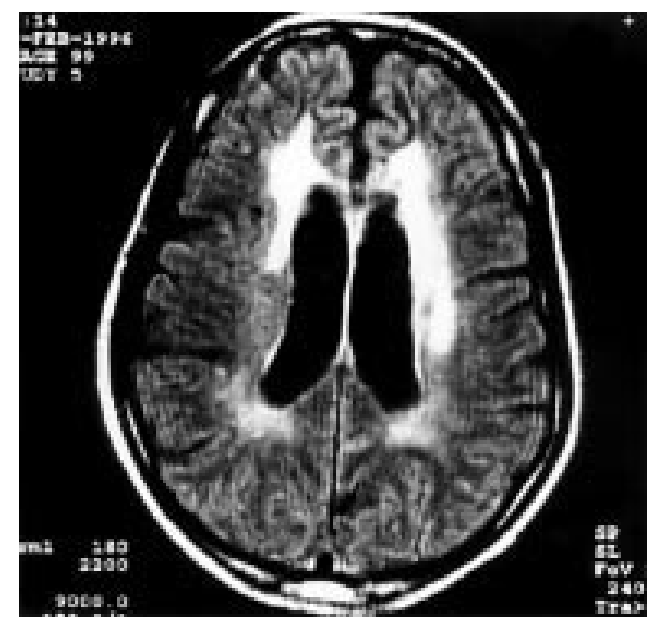

Figure 3 Illustrative MRI of the brain (case 1) showing extensive and confluent periventricular white matter hyperintensities in a FLAIR sequence.

present, was in excess of that described for MS. With respect to the MRI findings, our patients had supratentorial lesions that resemble those found in patients with MS. There was, as shown in the figures, involvement of the periventricular white matter, the centrum semiovale, the corona radiata, and the corpus callosum in patients 1,2 , 5, 6, 7, and 8. Considering the multiphasic course in these patients, these MRI findings in isolation would then be compatible with a diagnosis of MS. In patients $2,3,4,5,6,7$, and 8 there were spinal lesions identified by MRI. These were large and extended over several segments of the spinal cord in each case. In MS spinal lesions are usually small and extend over a single segment of the cord. ${ }^{12}$ The spinal MRI lesions in our patients were therefore in keeping with those described in ADEM or NMO. The cases described herein are from a population in which MS is rare. ${ }^{14}$ To date there are only 12 reported black patients from South Africa and Zimbabwe with possible MS. ${ }^{15}$

It follows from this discussion that the patients described herein do not have MS, ADEM or NMO but rather a disorder that interfaces all three conditions. The nature and topography of the white matter lesions on MRI in our patients suggest that the pathological processes in these patients is the same or similar to that of MS, ADEM, and NMO. Multiple sclerosis, ADEM, and NMO are thought to represent different ranges of a similar, if not the same, pathological process. ${ }^{16}$ Our patients provide support for this contention, which would then imply that they have a new and different or distinct phenotypic expression of this pathological process.

Demyelinating diseases of the CNS occur as a result an interaction between the environment and genes. In MS this results in an episodic inflammatory response whereas in ADEM an acute monophasic inflammatory demyelination occurs. The patients described by us have a different genetic background and a different environment to that described for MS. It is therefore not surprising that the phenotypic expression of CNS demyelination in these patients is different to MS.

The patients described here thus represent a new phenotypic expression of a recurrent (multiphasic), steroid-sensitive, inflammatory demyelinating disorder of the CNS occurring in black South Africans. The disorder is either a distinct inflammatory demyelinating disorder of the CNS of as yet unknown aetiology, or a varied form of MS (ADEM/NMO) occurring in a population (where the genetic trait and environmental factors for MS do not exist) with a low risk for MS.

1 Adams RD, Victor M. In: Barry BK, Schwarz M, Scott EJ, eds. Principles of neurology. 3rd ed. New York: McGraw-Hill, eds. Princ.

2 Ames FR, Louw S. Multiple sclerosis in coloured South Africans. F Neurol Neurosurg Psychiatry 1977;40:729-35

3 Cosnett JE. Multiple sclerosis and neuromyelitis optica. $S$ Afr Med f 1981;60:249-51

4 Weinshenker BG. The natural history of multiple sclerosis: a geographically based study, 1: clinical course and disability. Brain 1989;112:133-46.

5 Lublin FD, Reingold RC. Defining the clinical course of multiple sclerosis: results of an international survey. Neurology 1996;46:907-11

6 Behan PO, Moore MJ, Lisak RP. Acute disseminated encephalomyelitis. British fournal of Clinical Practice 1974;28:243-5.

7 Atlas SW, Grossman RI, Goldberg HI, et al. MR diagnosis of acute disseminated encephalomyelitis. $\mathcal{F}$ Comput Assist Tomogr 1986;10:798-801.

8 Kesselring J, Miller DH, Robb SA, et al. Acute disseminated encephalomyelitis MRI findings and the distinction from multiple sclerosis. Brain 1990;113:291-302.

9 Finsterer J, Grass R, Mamoli B. Immunoglobulins in acute, parainfectious, disseminated encephalo-myelitis. Clin $\mathrm{Neu}$ ropharmacol 1998;21:258-61.

10 Straub J, Chofflon M, Delavelle J. Early high dose intravenous methylprednisolone in acute disseminated encephalomyelitis: a successful recovery. Neurology 1997; 49:1145-7.

11 O'Riordan JI, Gomez-Anson B, Moseley IF, et al. Long term follow-up of patients with post-infectious encephalomyelitis: evidence for a monophasic disease. $\mathcal{F}$ Neurol Sci 1999;167:132-6.

12 O'Riordan JI, Gallagher UL, Thompson AJ, et al. Clinical, CSF and MRI findings in Devics neuromyelitis optica. $\mathcal{F}$ Neurol Neurosurg Psychiatry 1996;60:382-7.

13 Wingerchuk DM, Hogancamp WF, O'Brien PC, et al. The clinical course of neuromyelitis optica (Devic's syndrome). Neurology 1999;53:1107-14.

14 Poser C. The epidemiology of multiple sclerosis: a general overview. Ann Neurol 1994;36(S2):S180-93.

15 Dean G, Bhigjee AIG, Bill PLA, et al. Multiple sclerosis in black South Africans and Zimbabweans. $\mathcal{F}$ Neurol Neurosurg Psychiatry 1994;57:1064-9.

16 Kepes JJ. Large focal tumor-like demyelinating lesions of the brain: intermediate entity between multiple sclerosis and acute disseminated encephalomyelitis? A study of 31 patients. Ann Neurol 1993;33:18-27. 